\title{
Orthopedic surgery related infections; study of the importance of associated comorbidities in relationship with septic evolution
}

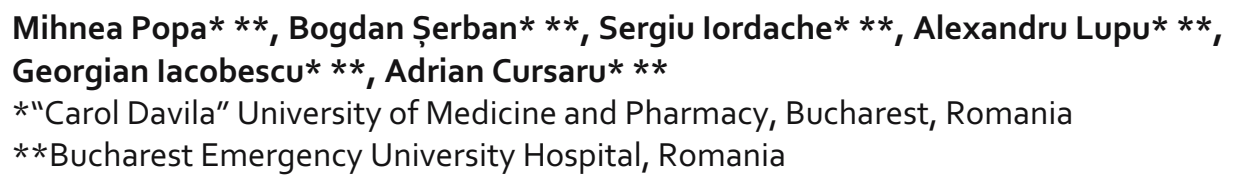

Correspondence to: Mihnea Popa, "Carol Davila" University of Medicine and Pharmacy, Bucharest, 8 Eroii Sanitari Blvd., District 5, Code 050474, Bucharest, Romania, Mobile phone: +40745809 582, E-mail: mihnea.ioan.popago@gmail.com

\begin{abstract}
The current socio-economic development has improved the standard of living of the general population. Technological evolution has led to the use of an increasing number of motor vehicles. Associated with the growing violence observed globally, orthopedic surgery involves frequent use of osteosynthesis materials and prostheses. Therefore, an increasing number of patients live with these implants, having a higher risk, compared to the general population, to develop complications. Although technological developments have increased the quality of life, it must not be forgotten that these foreign materials come with certain risks, the most feared being the associated infections.

We conducted an epidemiological study on infections identified in patients treated in the Orthopedics and Traumatology Department of Bucharest Emergency University Hospital, from 01.01.2016 to 01.06.2019. We were able to extract data about patients without neglecting their confidentiality.

The information sought, primarily involved the pathogen causing the infection, but at the same time we tried to build a more accurate history of each patient in order to determine the cause of infection and the risks posed by various associated pathologies. During these 4 years we were able to identify 139 patients who suffered an infection in the musculoskeletal system. In most cases we identified mono-microbial infections, but there were 7 cases in which two pathogens were identified.
\end{abstract}

Keywords: orthopedics and traumatology, infections, associated pathologies

\section{Introduction}

The current socio-economic development has improved the standard of living of the general population. Technological evolution has led to the use of an increasing number of motor vehicles. Associated with the growing violence observed globally, it is not surprising 
that the number of polytraumatic pathologies is increasing resulting in a rise in the number of musculoskeletal injuries [1,2].

When traumatic energy of a certain intensity is applied to a segment of the human body, depending on its momentum, it can cause injuries from the simplest contusions to significant tissue and bone destruction. Open fractures have poor prognosis in some cases, as the risk of infection can reach up to $30 \%$, thus being responsible for high mortality and morbidity rates [3-6].

Staphylococcus aureus is the most frequently isolated microorganism in infections of the musculoskeletal system (approximately $20-30 \%$ of all infections) $[7,8]$. Enterococci and streptococci are Gram-positive cocci identified in a proportion of 3-7\% and 1$10 \%$ respectively. Infections with Pseudomonas aeruginosa or other Gramnegative bacilli, such as enterobacteria, can occur in a proportion of $6-17 \%$, but numerous recent studies have described many cases of infectious pathology in which the etiological agents could not be identified (5-34\%), these findings raised a question mark, emphasizing the need to implement new high-performance microbiological diagnostic techniques [9-11].

It is necessary to identify the pathogen, followed by effective antibiotic therapy. The bactericidal concentration must be high on the surface of the implanted material, in the organic structures and in the bone tissue. The antibiotic must act on both dormant colonies and bacterial cells in the biofilm, without having adverse effects on the patient $[7,12,13]$.

Currently, substances with a bactericidal role are applied during surgery, having the advantage of achieving a useful local antibiotic concentration, without general effects on the host. The debut was the preparation of acrylic cement containing gentamicin in the 1970s, becoming a routine practice $[\mathbf{9 , 1 4 , 1 5 ]}$. The use of silver ions on the surface of implantable materials [16], the application of antimicrobial peptides to the surface of an implant [17], or the use of anti-biofilm dispersant [18], are other ways to address this issue.

A problem in the specialty of orthopedics and traumatology is the lack of a universal consensus on how to describe infections in the osteo-articular sphere. Many of the studies in the field use the guide of the Centers for Disease Control and Prevention (CDC), which describes 3 pathological entities depending on the depth of the infection (superficial and deep incisions, organ or space infections). However, this definition does not include osteomyelitis or infection of an implantable material. This problem becomes obvious when different specialized documents are studied. The technique of describing an infection may differ from one surgical team to another [19-21].

\section{Material and method}

We conducted an epidemiological study on infections identified in patients treated in the Orthopedics and Traumatology Department of Bucharest Emergency University Hospital, from 01.01.2016 to 01.06.2019. Using the hospital's patient management software, we were able to extract data about patients without neglecting their confidentiality. The information sought, primarily involved the pathogen causing the infection, but at the same time we tried to build a more accurate history of each patient in order to determine the cause of infection and the risks posed by various associated pathologies. Patients under the age of 18 were removed from the study and when one patient had several hospitalizations with the same pathology treated sequentially, the data were corroborated so that there was no artificially increased number of patients. After accumulating data for their interpretation, the software R ( $v$ 3.2.1) and RStudio ( $v$ 1.0.136) were used. 


\section{Results}

During these 4 years, we were able to identify 139 patients who suffered an infection in the musculoskeletal system. Monomicrobial infections were found in most cases, but there were 7 cases in which two pathogens were identified. According to literature, the most identified bacteria were from the genus Staphylococcus, followed by Enterobacteria and nonfermentative germs. 2 infections were also caused by enterococci.

The most frequently involved bacterium was Staphylococcus aureus (77 cases). In elderly patients with mobilization deficit and associated pathologies in the bronchopulmonary or urogenital sphere the following bacteria were identified: 16 strains of Klebsiella pneumoniae, 10 of Escherichia coli, 9 of Pseudomonas aeruginosa and 6 strains of Proteus mirabilis. In the case of polytraumatized patients who suffered accidents with high energies and who were hospitalized in the ATI section, strains of Serratia marcescens were isolated in 6 cases. Other types of microorganisms have been identified, but in a very small number, with little statistical significance (Fig. 1).

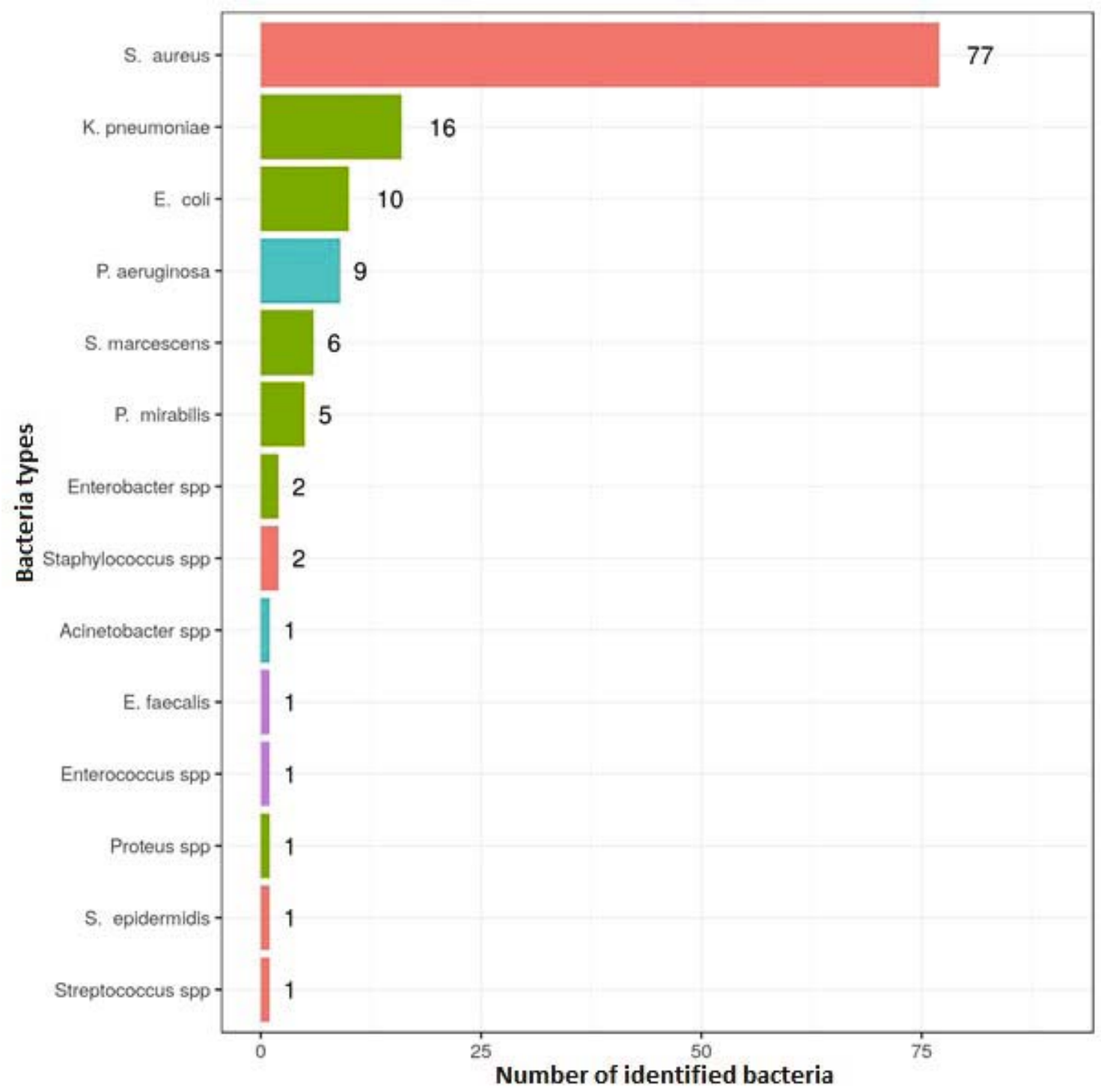

Fig. 1 Graphical representation of isolated bacterial strains, by gender, during the period 2016-2019 
In order to identify the causes that predispose to the appearance of an infection in the field of orthopedics and traumatology, we divided the cases into 4 categories, depending on the reason for hospitalization. Most of the young hospitalized patients were those who underwent surgery following a fracture, and in the case of the elderly, reintervention was needed to perform a revision of a septic arthroplasty. We were able to identify 11 septic arthritis caused by punctures or intra-articular infiltrations in patients without significant comorbidities. At the same time, we evaluated 23 cases of septic arthritis without surgery, occurring in immunocompromised patients with poor hygiene, superficial infections near the joint or in patients with diabetes and Charcot's foot (Fig. 2).

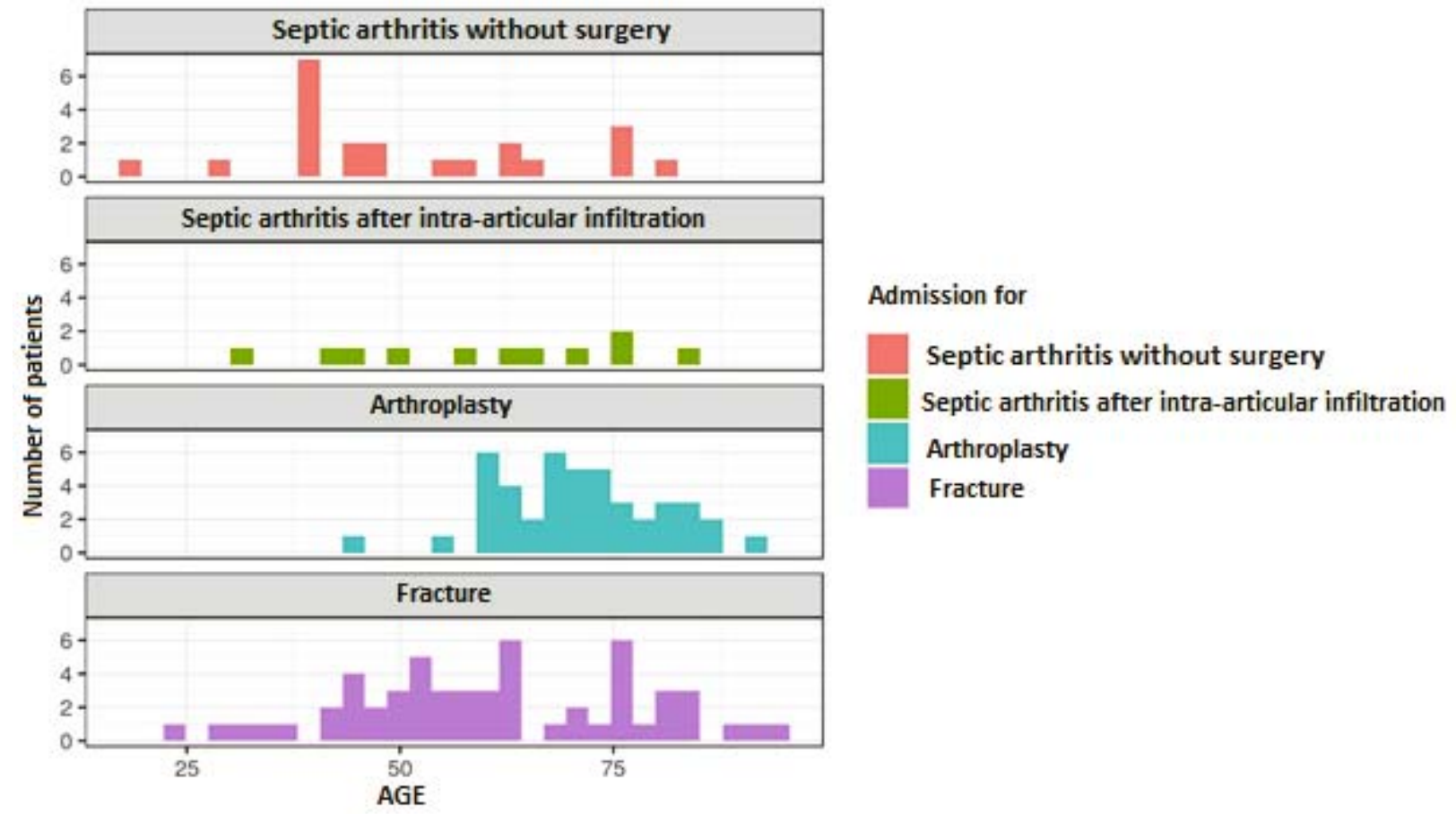

Fig. 2 Breakdown of the reason for hospitalization, for patients treated in Bucharest Emergency University Hospital, during the period 2016-2019

In our study, 57 patients were hospitalized on the orthopedic clinic of Bucharest Emergency University Hospital with a septic process related to the osteosynthesis material, implanted at an earlier time, when the patient suffered a fracture. Many of these patients were those who have been hospitalized as polytraumatized and who underwent surgery with different surgeons in more than one body segment, or those who have had open fractures for which, depending on the degree of soft tissue damage, osteosynthesis was performed per primam or an external fixator was fitted and then, at a later stage, the definitive osteosynthesis was performed. As observed in previous figures, most of the microbes involved were from the species Staphylococcus aureus and Klebsiella pneumonae, but also other microorganisms such as Proteus spp., Serratia marcescens and Pseudomonas aeruginosa (Fig. 3 ).

Infectious pathology related to septic arthritis triggered by intra-articular infiltration in conditions of deficient asepsis and antisepsis must be considered. The information extracted during the study showed 11 patients who suffered due to such minimally invasive procedures. Most of the identified pathogens were classified as $S$. aureus but there were also E. coli, Streptococcus spp. and Enterobacter spp. involved (Fig. 4). 


\section{Admission reason: fracture}

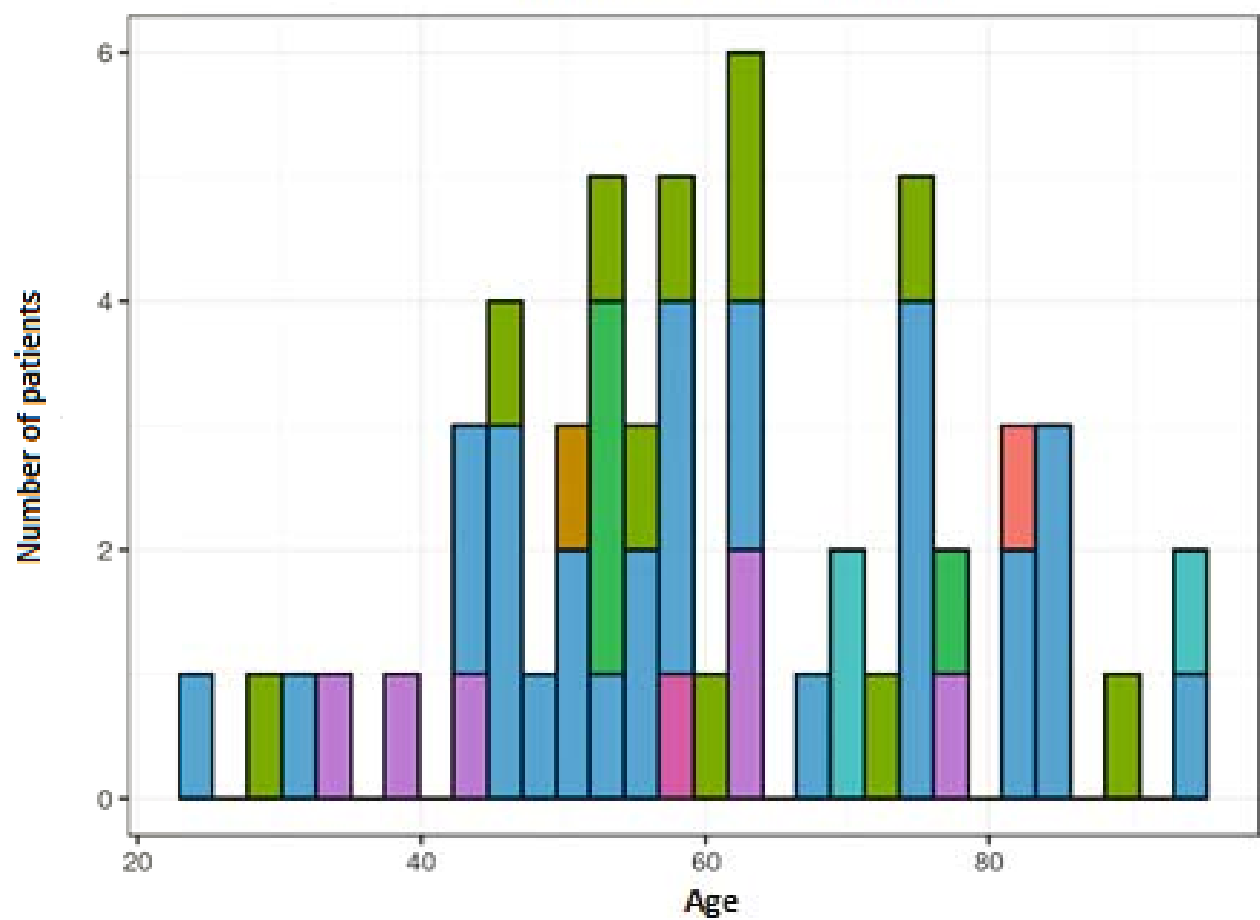

\section{Bacteria type}

E. coli

Entercbacter spp

K. pneumoniae

P. mirabelis

P. aeruginosa

S. aureus

S. marcescens

Staphylococcus spp

Fig. 3 Graphical representation of infectious agents involved in infections related to the pre-existence of a fracture, in relation to the age of patients, for strains isolated from patients admitted to Bucharest Emergency University Hospital, during 2016-2019

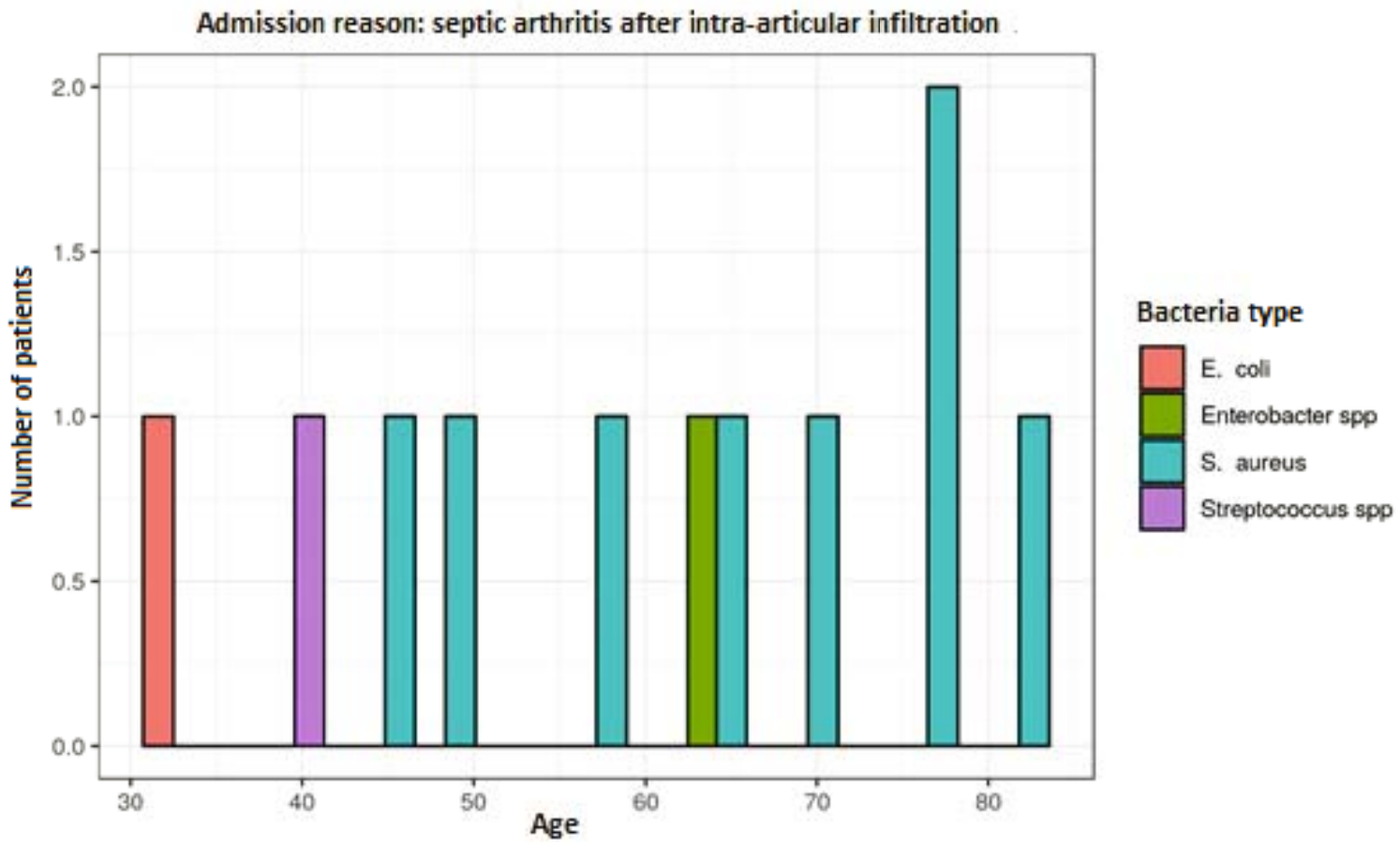

Fig. 4 Graphical representation of infectious agents involved in post-puncture septic arthritis according to patients' age, for strains isolated from patients admitted to Bucharest Emergency University Hospital, during 2016-2019 
After we conducted the study, we infection. Skin and especially therapeutically confirmed the statistical data presented in medical literature. The number of pathologies in the cardiovascular sphere (especially hypertension - hypertension and heart disease) was much higher, than other types of pathologies $[\mathbf{5}, \mathbf{1 2}, \mathbf{2 0}]$. Other problems were the presence of obesity, chronic smoking and type II diabetes, leading to an increased risk of neglected varicose veins led to a higher risk of infection for elderly patients who had at least one prosthetic implant. Patients suffering from hepatitis, with damage of the gastrointestinal system, had an increased risk of developing infections, in most cases with enterobacteria (Fig. 5).

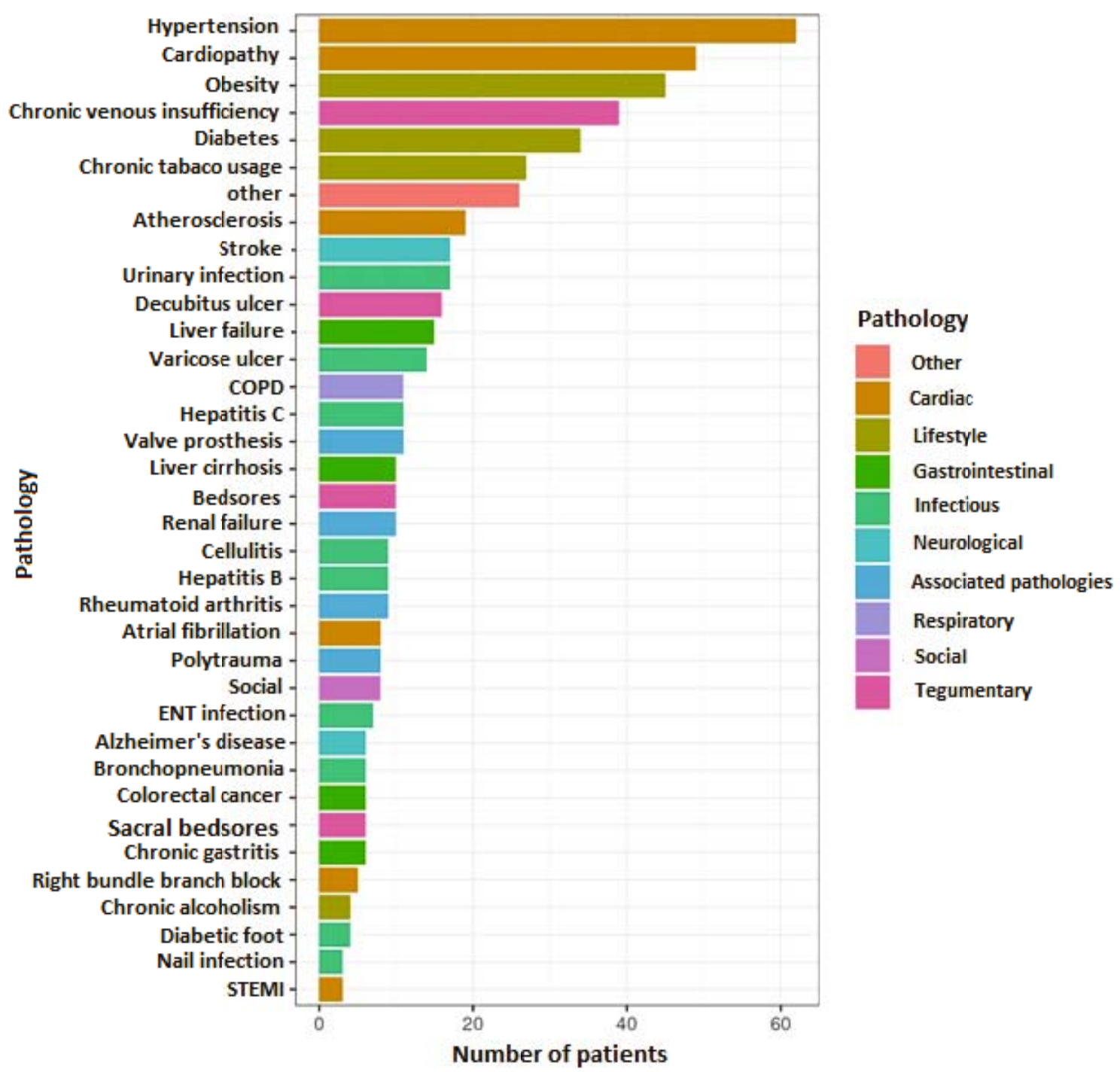

Fig. 5 Detailed graphic representation of the associated pathologies in the case of patients admitted to Bucharest Emergency University Hospital, during 2016-2019

\section{Discussions}

Meticulous surgical technique is an essential element, even more important in the case of polytraumatized patients. A method used in tandem with physical debridement is to irrigate injured areas with antiseptic solutions. CDC guidelines recommend the use 
of aqueous solutions based on iodine, but these studies are not performed on open fractures [22-24].

The most important aspect related to the post-operative management of the wound is related to the hygiene and aseptic technique applied by the auxiliary staff, when interacting with the patient $[\mathbf{1}, \mathbf{2 4}]$.

In the case of surgery, especially in traumatized patients, with blood loss before the operation, hypothermia can pose important problems. Patients in hypothermia have increased amounts of catecholamines in the blood that cause moments of hyperglycemia, as the temperature of these patients increases. Previously administered insulin may cause severe hypoglycaemia. Hypothermia affects blood circulation and can cause coagulopathies also influencing the migration of leukocytes, phagocytes, neutrophils and the production of cytokines leading to slowing of tissue recovery and the immune system $[\mathbf{1}, \mathbf{2 1}, \mathbf{2 5}, \mathbf{2 6}]$.

\section{Conclusions}

Infections in the field of orthopedics and traumatology are a side of this specialty that, without a rapid diagnosis and the establishment of a targeted treatment, can have disastrous effects, with implications for both patients and health systems. This type of pathology is not very well documented, the number of publications on this topic is relatively limited in both Romanian and international literature.

The information presented in this study is consistent with that in the literature both in terms of identified pathogens, their resistance, comorbidities and risk factors of patients with such pathology.

\section{Conflicts of interest}

Authors state no conflict of interest.
Informed Consent and Human and Animal Rights statements

An informed consent has been obtained from all individuals included in this study.

\section{Authorization for the use of human subjects}

Ethical approval: The research related to human use complies with all the relevant national regulations, institutional policies, is in accordance with the tenets of the Helsinki Declaration, and has been approved by the review board of Bucharest Emergency University Hospital, Romania.

\section{Acknowledgements}

None.

\section{Sources of Funding}

None.

\section{Disclosures}

None.

\section{References}

1. Azar FM, Canale ST, Beaty JH. Ed. Campbell's Operative Orthopedics. 4-volume Set. $13^{\text {th }}$, 2017, ed. Philadelphia, PA: Elsevier.

2. Cherian J, Lobo J, Ramesh L. A comparative study of Bacteriologica culture results using swab and tissue in open fractures: a pilot study. J Orthop Case Reports. 2019; 9(1):33-36.

3. Guerra M, Gregio F, Bernardi $A$ et al. Infection rate in adult patients with open fractures treated at the emergency hospital and at the ULBRA university hospital in Canoas, Rio Grande do Sul, Brazil. Rev Bras Ortop. 2017; 52(5):544-548.

4. Metsemakers W, Onsea J, Neutjens E et al. Prevention of fracture-related infection: a multidisciplinary care package. Int Orthop. 2017; 41:2457-2459.

5. Shao J, Zhang H, Yin B et al. Risk factors for surgical site infection following operative treatment of ankle fractures: A systematic review and meta-analysis. Int J Surg. 2018; 56:124-132.

6. Shao J, Chang $H$, Zhu $Y$ et al. Incidence and risk factors for surgical site infection after open reduction and internal fixation of tibial plateau fracture: A systematic review and meta-analysis. Int J Surg. 2017; 41:176-182.

7. Cooper R. Surgical site infections: epidemiology and microbiological aspects in trauma and orthopaedic surgery. Int Wound J. 2013; 10(suppl.1):3-8. 
8. Tande AJ, Patel R. Prosthetic joint infections. Clin. Microbiol Rev. 2014; 27:302-45.

9. Moriarty T, Kuehl R, Coenye T et al. Orthopaedic devicerelated infection: current and future interventions for improved prevention and treatment. Effort Open Rev. 2016; 1:89-99.

10. Krutz SM, Lau E, Schmier J et al. Infection burden for hipand knee arthroplasty in the United States. JArthroplasty. 2008; 23:984-91.

11. Hoiby $\mathrm{N}$, Bjarnshold T, Moser $\mathrm{C}$ et al. ESCMID guideline for the diagnosis and treatment of biofilm infections. Clin Microbiol Infect. 2015; 21(1):1-25.

12. Nguyen $S$, Robineau $O$, Titecat $M$ et al. Influence of daily dosage and frequency of administration of rifampicinlevofloxacin therapy on tolerance and effectiveness in 154 patients treated for prosthetic joint infections. Eur J Clin Microbiol Infect Dis. 2015; 34:1675-82.

13. Osmon DR, Berbari EF, Berendt AR et al. Diagnosis and management of prosthetic joint infection: clinical practice guidelines by the Infectious Diseases Society of America. Clin Infect Dis. 2013; 56:e1-e25.

14. Ter Boo GJ, Grijpma DW, Morirty TF et al. Antimicrobial delivery systems for local infection prophylaxis in orthopaedic and trauma surgery. Biomaterials. 2015; 52:113-25.

15. Nishitani K, Beck CA, Rosenberg AF et al. A diagnostic serum antibody test for patients with Staphylococcus aureus osteomyelitis. Clin Orthop Relat Res. 2015i 473:2735-49.

16. Brennan SA, Ni Fhoglu C, Devitt BM et al. Silver nanoparticles and their orthopaedic aplications. Bone Joint J. 2015; 97-B(5):582-9.

17. Wafa H, Grimer RJ, Reddy $K$ et al. Retrospective evaluation of the incidence of early periprosthetic infection with silver-treated endoprostheses in high-risk patients: case control study. Bone Joint J. 2015; 97B:2527.

18. Okshevsky M, Regina VR, Meyer RL. Extracellular DNA as a target for biofilm control. Curr Opin Biotechnol. 2015; 33:73-80.

19. Metsemakers W, Onsea J, Neutjens E et al. Prevention of fracture-related infection: a multidisciplinary care package. Int Orthop. 2017; 41:2457-2459.

20. Metsemakrs W, Kuehl R, Moriarty $T$ et al. Infection after fracture fixation: current surgical and microbiological concepts. Injury. 2018; 49(3):511-522.

21. Popa MI, Serban B, Lupu A, lacobescu G, Cursaru A. Orthopedic surgery related infections; Study of antibiotic resistance and associated comorbidities. ROJOST. 2020; 3(1):55-63.

22. Dancer S, Christison F, Eslami A et al. Is it worth screening elective orthopaedic patients for carriage of Staphylococcus aureus? A part-retrospective case-control study in a Scottish hospital. BMJ Open. 2016; 6(9):e011642.

23. Kasatpibal N, Whitney J, Dellinger E et al. Failure to redoes antibiotic prophylaxis in Long Surgery increases risk of Surgical site infection. Surg Infect. 2017; 18(4):474-484.

24. Mangram AJ, Horan TC, Pearson ML et al. Guideline for Prevention of Surgical Site Infection. Centers for Disease
Control and Prevention (CDC) Hospital Infection Control Practices Advisory Committee. Am J Infect Control. 1999; 27(2):97-132.

25. Popa Mihnea G, Ene R, Popa V et al. Posibilitatea diagnosticului infecțiilor ortopedice folosind microcalorimetria. Infectio.ro. 2018; 54(2):32-35.

26. Popa Mihnea G, Panti Z, Nica M et al. Microcalorimetria - o metodă Cu potențial în diagnosticul infecțiilor ortopedice. Infectio.ro. 2017; 49(1):42-45. 